\title{
Modelo Georreferenciado de Disponibilidade de Internet para Educação Superior Online: Estudo de Caso no Estado do Pará
}

\author{
Vitor Hugo M Gomes ${ }^{1}$, Rodrigo Dutra ${ }^{1}$, Weverson Nascimento ${ }^{1}$, Luiz C. Ferreira ${ }^{1}$ \\ Marcelino S. da Silva ${ }^{1}$, Aldebaro B. R. Klautau ${ }^{1}$, Antônio Pereira ${ }^{1}$ \\ ${ }^{1}$ Instituto de Tecnologia - Universidade Federal do Par a (UFPA) \\ Caixa Postal 479 - 66.075-110 - Belém - PA - Brasil \\ hugom4cedo@gmail.com, rodrigo.dutra@itec.ufpa.br, weverson@ufpa.br, \\ \{luizcf14,marcelino.silva\}@gmail.com, \{aldebaro, apereira\}@ufpa.br
}

\begin{abstract}
Due to social distancing measures implemented during the COVID19 pandemic, several higher education institutions are using internet applications to provide instructional content to their students. However, there is widespread inequality in access to these resources. In this work, we model the dispersion of the mobile Internet signal based on data of geolocation of cell towers, land cover assessment and altimetry to provide a clearer picture of students' access to remote learning. The results of our case study show that there is a large number of students from the Federal University of Pará whose address is outside the coverage area of mobile providers.
\end{abstract}

Resumo. Devido às medidas de distanciamento social implementadas durante a pandemia Covid-19, várias instituições de ensino superior estão usando aplicativos de Internet para fornecer conteúdo instrutivo aos seus alunos. No entanto, há uma desigualdade generalizada no acesso a esses recursos. Neste trabalho, modelamos a dispersão do sinal de Internet móvel com base em dados de geolocalização de torres de celular, avaliação de cobertura de terra e altimetria para fornecer uma imagem mais clara do acesso dos alunos à aula remota. Os resultados do estudo de caso mostram que há um grande número de estudantes da Universidade Federal do Pará cujo endereço está fora da área de cobertura dos provedores de telefonia móvel.

\section{Introdução}

De acordo com a Organização das Nações para a Educação, Ciência e Cultura (UNESCO) [Zelezny-Green et al. 2018], a inclusão digital tem como objetivo impulsionar e democratizar o acesso às tecnologias de informação e a comunicação. Entretanto, cerca de 6 bilhões no mundo ainda não possuem acesso à Internet de banda larga, 4 bilhões à Internet convencional e 2 bilhões à telefone móvel [Zelezny-Green et al. 2018].

A inclusão digital permite o acesso a uma gama de serviços públicos e privados, proporcionando o acesso a coleções de bibliotecas, acesso à educação, interação social, entretenimento digital e assistência médica, [Fanea-Ivanovici and Pană 2020]. Entre as barreiras à inclusão digital poderíamos listar: i) falta de infraestrutura, ii) custos de acesso e acessibilidade, iii) falta de instrução e iv) motivação sociocultural. No Brasil, a precariedade do acesso à internet nas escolas públicas forma uma quinta barreira [Fernandes et al. 2020]. 
Desde o primeiro caso oficial relatado no final de 2019, a Covid-19 fraturou a rotina de bilhões de pessoas em todo o mundo [Xiong et al. 2020]. Especialmente depois que a OMS declarou estado de pandemia global devido ao aumento do número de casos da doença e o conhecimento de seu modo de transmissão, vários países implementaram políticas para restringir a mobilidade e o contato social para evitar a sobrecarga de seu sistema de saúde.

A nova realidade imposta pela pandemia levou à adoção de estratégias de educação remota usando aplicativos de Internet, como Zoom®e Google Meet ${ }^{\circledR}$. No entanto, a aprendizagem remota depende do acesso confiável e rápido à internet, que é uma realidade distante para a maioria dos estudantes brasileiros, especialmente aqueles matriculados em instituições públicas, do ensino fundamental à universidade

Problemas como a disponibilidade de uma conexão à Internet são recorrentes em locais remotos do globo, com ofertas de serviços precárias ou até inexistentes. Dados do Ministério da Educação [MEC 2020], por exemplo, mostram que 63\%dos estudantes que se submeteram ao Exame Nacional do Ensino Médio (Enem) em 2020 não tinham acesso à internet.

Uma forma de contribuir para essa questão é mapear a acessibilidade de universitários em situação de aprendizagem remota à rede de telefonia móvel. Essa questão é análoga ao mapeamento georreferenciado da disponibilidade de recursos naturais (como fontes de água, alimentos, etc.) em estudos ecológicos e de fontes sonoras nas cidades [Aumond et al. 2018], e risco de infecção [Abdrakhmanov et al. 2017].

Apesar da popularização do uso da Internet no Brasil, o acesso ainda é muito restrito principalmente nas áreas periféricas de grandes centros urbanos e áreas rurais com muitos pontos cegos onde o acesso é negado. Saber quantos alunos podem acessar a Internet ajuda no planejamento de estratégias para implantação de um ensino a distância mais eficiente. Uma forma de obter essa estimativa é mapear os locais que são atendidos pelas estações de rádio base (ERB) que fornecem acesso à Internet e identificar alunos que estão ao alcance da ERB individual. Esse tipo de análise pode ser realizada com o Google Earth Engine (GEE), uma plataforma em nuvem que usa imagens de satélite e informações de geolocalização [Amani et al. 2020].

O artigo a seguir é organizado da seguinte forma: a Seção 2 apresenta os trabalhos relacionados, Seção 3 apresenta a metodologia adotada, a Seção 4 os resultados e, por fim, as conclusões do trabalho são apresentadas na Seção 5.

\section{Trabalhos Relacionados}

O trabalho de [Ratusznei et al. 2016] apresenta uma avaliação de desempenho sobre o consumo de vídeo em praças de São Paulo, utilizando métricas como QoS e QoE para monitoramento de qualidade durante a execução de aplicativos streaming. De acordo com o estudo, algumas praças permitem visualização com qualidade adequada com codificação de até $1200 \mathrm{Kbps}$.

O trabalho de [Godoi et al. 2020] visa analisar o ensino remoto nos cursos de ensino superior em educação física, identificando os desafios e as aprendizagens dos professores universitários. Os resultados sugerem que a pandemia de Covid-19 potencializou o desenvolvimento profissional dos professores, oportunizando novas aprendizagens rela- 
cionadas à integração das Tecnologias Digitais de Informação e Comunicação (TDICs).

O estudo realizado por [Leão et al. 2019] demonstra a experiência de uso das Tecnologias da Informação e Comunicação (TICs) e redes comunitárias nos municípios de Boa Vista - Acará - Pará e Campo Verde - Concórdia do Pará. Utilizando backhaul com liberação de pontos de Wi-Fi na escola da comunidade. O trabalho relata a dificuldade em diminuir a fronteira digital naquela região por conta das distâncias que essas comunidades estão dos centros urbanos em meio a margem de rios na grande dimensão territorial da região amazônica.

Já o trabalho de [Azubuike et al. 2021], tem como objetivo avaliar o impacto da Covid-19 no acesso a educação da Nigéria. Diferentemente do presente trabalho, este estudo foi realizado de pesquisa através de entrevistas em 2020 com uma população de 1901 pessoas, dentre estes 554 estudantes distribuídos por 31 estados das 6 regiões da Nigéria. Os estudantes que estavam estudando remotamente foram perguntados sobre os problemas sofridos nesse período, $38 \%$ apresentaram problemas relacionados a créditos de telefone e dados de Internet e outros $8 \%$ reportaram apenas a internet como fonte de problemas para a conexão, ressaltando assim o problema ocasionado por falta de condições financeiras e de internet para o estudo remoto.

Assim como [Azubuike et al. 2021], o estudo de [Rotas and Cahapay 2020] elenca as dificuldades vividas pelos estudantes no período de isolamento social ocasionado pela Covid-19, porém dessa vez focando no cenário das Filipinas. Esse trabalho conduziu uma pesquisa qualitativa utilizando formulários online. Um dos problemas elencados por alunos que moram em áreas remotas foi a falta ou o mal sinal de Internet, forçando alguns estudantes a trajetos de 20 minutos só para ter acesso de internet para o ensino remoto.

Por fim, o estudo de [de Castro et al. 2018] tem como objetivo implementar de forma prática uma rede de dados na comunidade de Boa Vista do Acará, utilizando uma rede de dados fornecida pela UFPA para atender pontos estratégicos da comunidade: escolas, posto de saúde e o posto policial. O estudo utiliza o software pathloss 4.0 para projetar a rede e analisar a viabilidade do link.

Além de realizar uma análise específica para o Estado do Pará, o presente trabalho possui como diferencial, a utilização da geolocalização dos estudantes a partir de cálculos, medidas e parâmetros objetivos para identificar os alunos que estão fora da área de cobertura das redes celulares.

\section{Metodologia}

A área de estudo abrange o território do estado do Pará, o segundo maior estado da federação localizado no norte do Brasil, com uma área de $1.245 .870,798 \mathrm{~km}^{2}$, com uma população estimada em 8.690.745 pessoas, e uma densidade demográfica de 6.07 $\mathrm{hab} / \mathrm{km} 2$, segundo o IBGE [Brasil 2010].

A Figura 1, ilustra a localização dos estudantes a partir de dados de coordenadas geográficas no estado do Pará. Neste estudo, foram identificados 27827 alunos que podem apresentar dificuldades de acesso à Internet. A seguir, serão descritos os métodos para identificação desses alunos. 


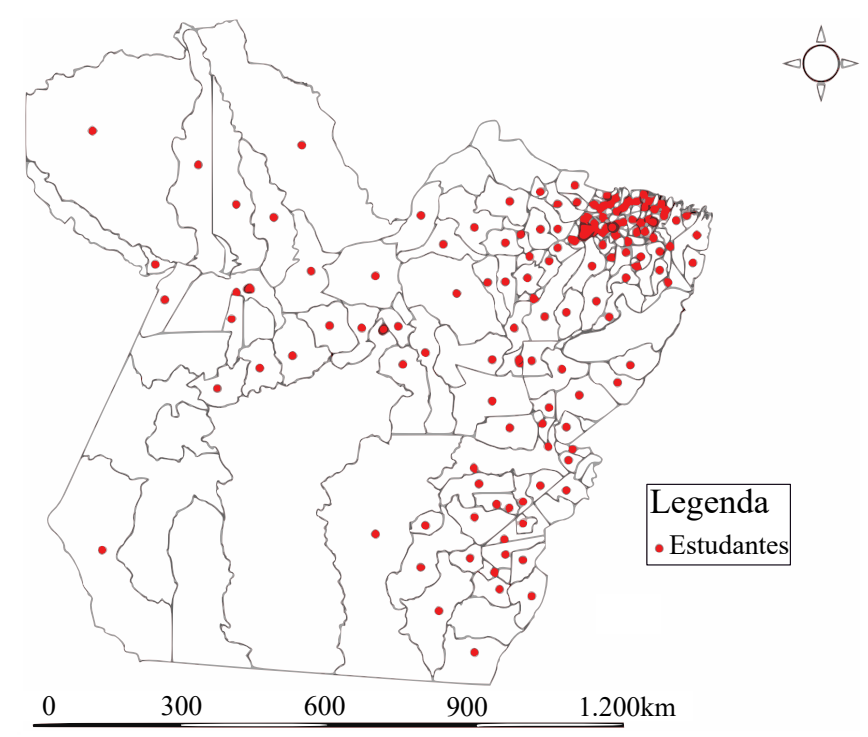

Figura 1. Local de residência dos alunos.

\subsection{Base de Dados}

Foram utilizados dois conjuntos de dados obtidos da Agência Nacional de Telecomunicações (ANATEL) e da UFPA. O banco de dados da ANATEL contém informações fornecidas pelas operadoras para obtenção de licenças operacionais e inclui o endereço, altura e frequência de funcionamento de sua ERB em todo o Brasil. Assim, assumindo que todas as ERBs licenciadas estejam em operação, foram selecionadas ERBs licenciadas no estado do Pará e os dados de endereço foram transformados em pontos de latitude e longitude utilizando a API Google-maps.

O banco de dados fornecido pela UFPA contém os CEPs fornecidos por cada estudante de graduação no momento de sua matrícula na universidade. Assim, assumindo que os alunos retornaram ao local registrado como CEP neste período de emergência, é possível estimar sua localização com base na latitude e longitude através da API googlemaps.

A localização georreferenciada dos alunos e das antenas ERBs foi inserida na plataforma GEE para análise do acesso dos alunos aos sinais de Internet durante esse período de emergência.

\subsection{Google Earth Engine (GEE)}

A plataforma possui um editor de código baseado em javascript que é utilizado para implementação de diferentes ferramentas de análises. Outra funcionalidade do GEE é o carregamento de dados geoespaciais, base de dados e imagens. Os arquivos contendo shapefile do estado do Pará, base de dados contendo as informações sobre alunos, e informações sobre as ERBs serão importados para o sistema com o comando assets. Em seguida, após a importação desses dados, utilizamos o editor de códigos para implementação da equação que será utilizada para medir a perda de sinal.

\subsection{Path loss (PL)}

Para estimar se o estudante está na área de cobertura de uma ERB, primeiro calculou-se a atenuação do sinal. Neste estudo, o modelo escolhido foi o COST 231 - HATA, desenvol- 
vido pela Cooperação Europeia em Ciência e Tecnologia que abrange frequências entre 1500 e $2000 \mathrm{MHz}$, antenas da estação móvel de 1 a 10 metros de altura, antenas ERB de 30 a 200 metros de altura e distâncias de ligação entre 1 e $20 \mathrm{Km}$. O modelo COST 231 HATA também permite a parametrização em sua modelagem matemática com o objetivo de abranger ambientes urbanos, suburbanos e rurais [Akhpashev and Andreev 2016]. O cálculo de perda de caminho é expresso de acordo com a equação 1:

$P L_{(d B)}=46,3+33,9 \log \left(f_{c}\right)-13,82 l \log \left(h_{t e}\right)-a\left(h_{m}\right)+\left[44,9-6,55 \log \left(h_{t e}\right)\right] \log (d)+C$.

Onde:

- $P L_{(d B)}$ : É a perda total do caminho em [dB]

- $f_{c}=1500$ : Frequência [Mhz]

- $d=1$ : Distância [m]

- $h_{m}=5$ : Altura da antena móvel [m]

- $a=170$ : Correção de altura da estação de celulares

- $h_{t e}=60:$ Altura da ERB

- $C=0$ : Coeficiente $=0$, é usado em cidades de médio porte e áreas suburbanas

Para cada foi, calculada o Path Loss entre o mesmo e as ERBs. Os resultados foram utilizados para estimar a potência do sinal recebido de acordo com o modelo descrito na próxima seção.

\subsection{Power of Referral Signals Received (RSRP)}

O parâmetro RSRP é um conjunto de medições da camada física do E-UTRAN Node B (eNodeB), que é realizado entre o equipamento do usuário (UE) e o eNodeB. O usuário se conecta com seu equipamento no eNodeB mais próximo onde os valores variam entre -140 e $-44 \mathrm{dBm}$. Este parâmetro é um dos principais utilizados na arquitetura LTE para a seleção do eNodeB mais próximo da UE. As medições de RSRP foram mapeadas em um intervalo que varia de 0 a 97 [Awad and Mkwawa 2017, Nofitasari and Haryadi 2017].

$$
R S R P=R S S I-10 \log (12 * N)
$$

Onde:

- RSRP: Potência do Sinal de Referência Recebido [dBm]

- RSSI: (Indicador de força do sinal recebido) é a força de sinal recebido por um usuário em uma faixa específica de frequência, incluindo ruído e interferência $[\mathrm{dBm}]$

- $N$ : Número do bloco de recursos usado por OFDMA

\subsection{Mapbiomas}

Para a implementação do modelo de previsão de cobertura de sinal nas diversas comunidades da zona rural e aglomerados urbanos no Estado do Pará, utilizamos mapas da região obtidos através do projeto MapBiomas. A obtenção do mapa do brasil em formato .tiff, foi feita através de download no ano de 2019 e importado para a plataforma GEE, onde a delimitação do território no mapbiomas foi feito através de recorte utilizando o método clip() que recebe como argumento o shapefile do território do estado do Pará. 
Tabela 1. Parâmetros RSRP, adaptado de [Awad and Mkwawa 2017]

\begin{tabular}{|l|l|l|}
\hline RSRP & Força do Sinal & Descrição \\
\hline$>=-80 \mathrm{dBm}$ & Excelente & Sinal Forte \\
\hline$-80 \mathrm{dBm}$ até $-90 \mathrm{dBm}$ & Bom & Boa Velocidade \\
\hline$-90 \mathrm{dBm}$ até $-100 \mathrm{dBm}$ & Rasoável $\backslash$ Pobre & $\begin{array}{l}\text { Dados podem ser perdidos à medida } \\
\text { que se aproxima de }-100 \mathrm{dBm}\end{array}$ \\
\hline$<=-100 \mathrm{dBm}$ & Sem sinal & Perda de conexão \\
\hline
\end{tabular}

\subsection{Qgis}

Para análise de dados, utilizou-se o banco de dados que foi exportado anteriormente através da plataforma GEE. A escolha do programa Qgis 3.1 ocorreu em função da eficácia de diferentes estudos e a existência de ferramentas para análise vetorial de forma simplificada. Outro fator que pesa na escolha da ferramenta é que se trata de software livre. Dessa forma, foi utilizado um arquivo de imagem do estado do Pará, recortado em formato .tiff, contendo as informações do Projeto MapBiomas, também exportadas da plataforma GEE, para então, adicionar camadas com coordenadas geográficas dos alunos e ERBs no estado do Pará dando prosseguimento às análises.

\section{Resultados}

Para um total de 27827 alunos, foram identificados 9052 ou 32,5\% de alunos que não possuem meios de acesso à Internet em todo o estado. Deste modo, foi realizado uma análise vetorial para obter a distância em que se encontram da ERB mais próxima para a identificação das possíveis soluções.

A tendência central em relação à distribuição aponta que, em média, os alunos estão à cerca de 19,1 km da ERB mais próxima. Da mesma forma, foram tomadas medidas de distância entre os alunos e o campus mais próximo da instituição de ensino, a fim de facilitar o acesso à infraestrutura necessária para o exercício das atividades acadêmicas utilizando meios de acesso às informações da instituição. O resultado revela que, em média, os alunos estão à $57 \mathrm{~km}$ do campus mais próximo, cabendo análise por parte dos gestores sobre a viabilidade de disponibilizar essa infraestrutura para aqueles que podem acessá-la com segurança para o exercício das atividades.

A partir de dados do uso da terra fornecidas pelo projeto MapBiomas. Foi possível realizar medições e cálculos através dos parâmetros RSRP para identificar alunos fora da área de cobertura das redes celulares e, assim, apresentar propostas de infraestruturas que podem ser utilizadas para atender tais alunos.

\subsection{Expansão do Sinal 4G em Áreas Urbanas ou Próximas das ERBs (Solução 1)}

Ainda que os alunos estejam bem próximos à uma ERB ou dentro da área de cobertura, é interessante medir a qualidade do sinal recebido pelo UE. Nesse contexto, os resultados obtidos pelo parâmetro RSRP, utilizados neste estudo, identificou alunos que mesmo estando relativamente próximo à uma ERB ainda podem apresentar dificuldades de acesso. Características como o grande número de edifícios, árvores, relevo acidentado e distância da ERB podem servir como um obstáculo a propagação do sinal eletromagnético. 
A adoção de políticas que promovam a aquisição de equipamentos, além da distribuição de chips com internet ilimitada, certamente contribuirá significativamente para a inclusão digital em um momento em que a educação está lado a lado com meios digitais de acesso à informação. Porém, como já descrito, muitos alunos estão fora da área de cobertura das operadoras celulares e, desta forma, é necessário que equipamentos sejam utilizados para amplificação do sinal nas áreas próximas de ERBs que possuem problemas de recepção de sinal muitas vezes ocasionados por edificações (cenário urbano) ou por densa e alta vegetação (cenário rural típico da em questão). Para esta solução, tabela 2 descreve o conjunto de equipamentos principais com preço médio por unidade.

Tabela 2. Custo dos Principais Equipamentos na Solução 1.

\begin{tabular}{|c|c|c|l|}
\hline Equipamentos & Quant. & Valor Unit. (R\$) & \multicolumn{1}{|c|}{ Observação } \\
\hline Amplificador 4G & 1 & 674,53 & $\begin{array}{l}\text { Possibilita alto ganho para } \\
\text { acesso à Internet por meio } \\
\text { de uma operadora de } \\
\text { telefonia }\end{array}$ \\
\hline Cabo de rede cat5 & $100 \mathrm{~m}$ & 100,00 & $\begin{array}{l}\text { Possibilita a conexão entre } \\
\text { o roteador externo, POE e } \\
\text { roteador wifi }\end{array}$ \\
\hline Roteador wifi & 1 & 162,90 & $\begin{array}{l}\text { Permite distribuir o sinal de } \\
\text { Internet }\end{array}$ \\
\hline Antena externa 22dBi & 1 & 103,10 & $\begin{array}{l}\text { Possibilitaa melhoria da } \\
\text { recepção de sinal em regiões } \\
\text { com sinal baixo }\end{array}$ \\
\hline
\end{tabular}

\subsection{Internet comunitária em localização remota (Solução 2)}

Segundo a Agência Nacional de Desenvolvimento Da América Latina (ANATEL), alguns países da América Latina, incluindo o Brasil, já possuem diretrizes para a regulamentação do Private Limited Service (SLP). No Brasil, a Resolução nº 617/2013 trata das definições de autorização para uso do SLP. Vale ressaltar que o uso da autorização para SLP é dispensado para Internet comunitária, porém os equipamentos utilizados para acesso à Internet devem ser aprovados pela ANATEL.

Com relação à implementação da Internet comunitária, a própria comunidade pode iniciar a construção e planejar o modelo de negócio que seja melhor adéqua para o acesso à Internet nessas regiões remotas.

Na solução elaborada, sugere-se o uso de uma rede de Internet via rádio multiponto com rede de malha. Isso garante que os roteadores nas casas dos moradores possam transmitir o sinal, cobrindo uma área ainda maior. Em algumas comunidades, entretanto, pode haver indisponibilidade de sinal diretamente com o provedor de Internet. Para contornar esse problema é necessário usar uma segunda antena direcional em um ponto distante da comunidade para fornecer o link de Internet que será decodificado através da antena presente na comunidade [Blanco 2017].

Em um cenário real de implementação, a quantidade desses equipamentos podem mudar de acordo com o obstáculo imposto pela geografia local. A Tabela 3 descreve o conjunto dos principais equipamentos utilizados no cenário descrito nesta seção. 
Tabela 3. Custo dos Principais Equipamentos na Solução 2.

\begin{tabular}{|c|c|c|c|}
\hline Equipamentos & Quant. & Valor Unit. (R\$) & Observação \\
\hline $\begin{array}{l}\text { Antena direcional } \\
5,8 \mathrm{Ghz}\end{array}$ & 1 & $2.010,65$ & $\begin{array}{l}\text { Permite a conexão da antena } \\
\text { direcional com o link de } \\
\text { Internet }\end{array}$ \\
\hline $\begin{array}{l}\text { Antena setorial 16dbi } \\
5 \mathrm{ghz} 100 \mathrm{mbps}+\mathrm{ac}\end{array}$ & 1 & 850,00 & $\begin{array}{l}\text { Permite a distribuição de sinal } \\
\text { de Internet na comunidade }\end{array}$ \\
\hline RouterBoard 750Gl & 1 & 530,00 & Gerenciamento de Rede \\
\hline $\begin{array}{l}\text { CPE AC 23dbi 100+ } \\
\text { mbps }\end{array}$ & 1 & 609,90 & $\begin{array}{l}\text { Antenado usuário para } \\
\text { captação de sinal }\end{array}$ \\
\hline $\begin{array}{l}\text { Cabo de rede blindado } \\
\text { Cat5 }\end{array}$ & - & - & Conexões do usuário \\
\hline $\begin{array}{l}\text { Roteador Wireless Dual } \\
\text { Band }\end{array}$ & 1 & 368,90 & $\begin{array}{l}\text { Responsável por retransmitir } \\
\text { o sinal das antenas setoriais } \\
\text { para os dispositivos. Pode ser } \\
\text { utilizado para retransmissão } \\
\text { de sinal em rede mesh }\end{array}$ \\
\hline
\end{tabular}

\subsection{Aplicação dos custos das soluções propostas}

Por meio deste estudo, foram identificados duas soluções que podem auxiliar os alunos que, devido ao isolamento social causado pela pandemia Covid-19, estão tendo dificuldades para continuar seus estudos. Para uma melhor distribuição desses recursos, as distâncias entre os alunos e a ERB mais próxima devem ser levadas em conta. Quantificamos a seguir os alunos que podem ser atendidos pela solução 1, que é mais econômica e solução 2, que busca resolver problemas de quem vive em comunidades mais isoladas. Como mostrado na Figura 2.

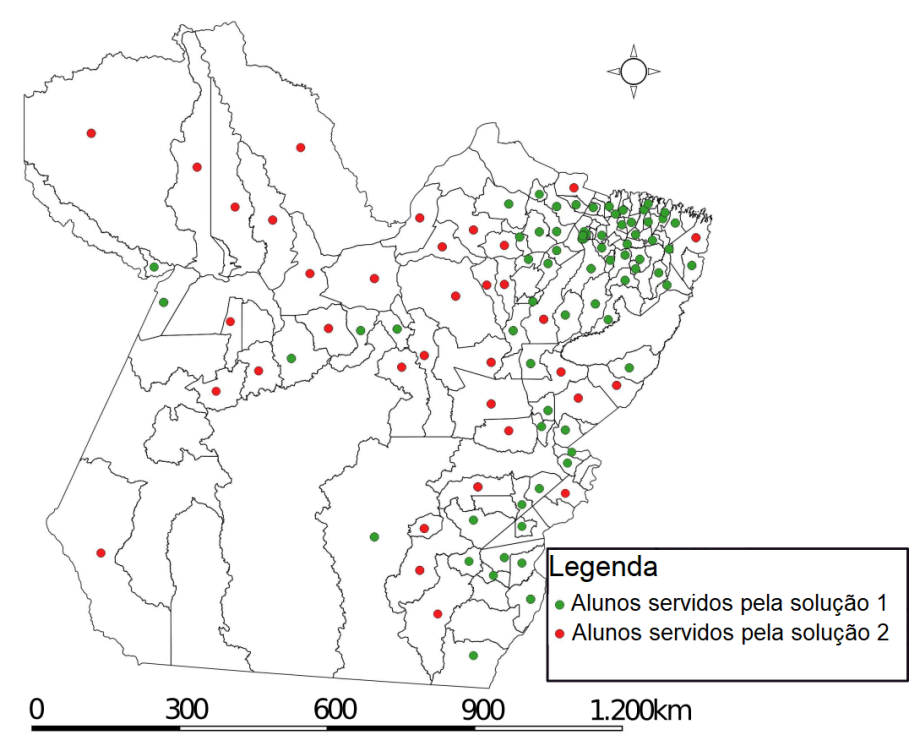

Figura 2. Distribuição geográfica dos alunos atendidos pelas duas soluções.

A Figura 2, ilustra a distribuição em todo o território do estado do Pará dos alunos que podem ser atendidos pelas soluções 1 e 2 que, somados, resultam em um total 
de 9052 estudantes que de alguma maneira não possuem acesso integral aos meios digitais. Na solução 1, foram contabilizados 6398 alunos que estão a uma distância de aproximadamente $25 \mathrm{~km}$ da ERB mais próxima. Deste modo, o custo total seria de R\$ 6.657.310,94, aproximadamente. Na solução 2, foram contabilizados 2654 alunos que estão a uma distância acima de $25 \mathrm{~km}$ da ERB mais próxima, sugerindo uma solução um pouco mais complexa para obtenção do sinal eletromagnético, com o custo de $\mathrm{R} \$$ 11.596.520,30, aproximadamente.

\section{Conclusão}

A pandemia de Covid-19 escancarou o sério problema de acessibilidade com relação ao uso das Tecnologias da Informação e Comunicação TICs no Brasil, afetando diversos setores, inclusive, os alunos de diversas etapas de ensino. A proposta deste estudo teve como objetivo identificar a dimensão do problema de acessibilidade de ensino remoto dos estudantes da UFPA e propor meios de acesso que possam remediar os prejuízos causados pela adoção repentina das aulas por meio das TICs, que a partir deste momento estão se tornando requisitos ainda mais importantes para equidade na educação.

Os resultados obtidos por meio deste estudo, evidenciam que a quantidade numerosa de ERB em uma determinada região nem sempre significa acesso igualitário à informação por meio digital. A tendência é que boa parte dos alunos sem acesso à Internet estejam nas proximidades de uma ERB, denotando que o esforço necessário para que o aluno tenha acesso a informação por meio digital possa ser realizado por equipamentos de baixo custo, revelando a baixa complexidade para a resolução do problema. No entanto, vale ressaltar que, existe a possibilidade de que o UE que mesmo que o estudante esteja a uma distância aceitável para a decodificação do sinal da ERB, barreiras naturais podem impedir o acesso de qualidade.

Conforme descrito anteriormente, existem aqueles que estão localizados em regiões remotas e por consequência se tornam economicamente inviáveis por parte das operadoras para implementação de uma ERB. Deste modo, o incentivo a TICs de baixo custo administrada pelos próprios estudantes ou moradores do entorno é uma solução para promover a inclusão digital de toda a comunidade.

Além das questões de acessibilidade dos alunos, o uso das soluções propostas neste trabalho podem fomentar o desenvolvimento local como um todo, por exemplo, incluindo também estudantes do ensino básico e pequenos empreendedores a desenvolver seu próprio negócio movimentando a economia local. Criando uma consciência por parte da comunidade sobre os benefícios de estar incluído digitalmente.

\section{Referências}

Abdrakhmanov, S., Mukhanbetkaliyev, Y., Korennoy, F., Sultanov, A., Kadyrov, A., Kushubaev, D., and Bakishev, T. (2017). Maximum entropy modeling risk of anthrax in the republic of kazakhstan. Preventive Veterinary Medicine, 144:149-157.

Akhpashev, R. V. and Andreev, A. V. (2016). Cost 231 hata adaptation model for urban conditions in lte networks. In 2016 17th International Conference of Young Specialists on Micro/Nanotechnologies and Electron Devices (EDM), pages 64-66. IEEE.

Amani, M., Ghorbanian, A., Ahmadi, S. A., Kakooei, M., Moghimi, A., Mirmazloumi, S. M., Moghaddam, S. H. A., Mahdavi, S., Ghahremanloo, M., Parsian, S., et al. 
(2020). Google earth engine cloud computing platform for remote sensing big data applications: A comprehensive review. IEEE Journal of Selected Topics in Applied Earth Observations and Remote Sensing.

Aumond, P., Can, A., Mallet, V., De Coensel, B., Ribeiro, C., Botteldooren, D., and Lavandier, C. (2018). Kriging-based spatial interpolation from measurements for sound level mapping in urban areas. The journal of the acoustical society of America, 143(5):2847-2857.

Awad, N. and Mkwawa, I.-H. (2017). The impact of the reference signal received power to quality of experience for video streaming over lte network. In 2017 Annual Conference on New Trends in Information \& Communications Technology Applications (NTICT), pages 192-196. IEEE.

Azubuike, O. B., Adegboye, O., and Quadri, H. (2021). Who gets to learn in a pandemic? exploring the digital divide in remote learning during the covid-19 pandemic in nigeria. International Journal of Educational Research Open, 2:100022.

Blanco, Marcelo, R. M. e. R. N. (2017). Como montar e regularizar um provedor comunitário.

Brasil, I. (2010). Instituto brasileiro de geografia e estatística. Censo demográfico, 2010.

de Castro, L. B., da Silva Ferreira, S. K., and Boas, B. V. (2018). Projeto celcom: Uma solução de baixo custo para a inclusão digital e social em comunidades isoladas no brasil. SENID-5o Seminário Nacional de Inclusão Digital.

Fanea-Ivanovici, M. and Pană, M.-C. (2020). From culture to smart culture. how digital transformations enhance citizens' well-being through better cultural accessibility and inclusion. IEEE Access, 8:37988-38000.

Fernandes, S. M., Henn, L. G., and Kist, L. B. (2020). O ensino a distância no brasil: alguns apontamentos. Research, Society and Development, 9(2):19.

Godoi, M., Beraldo Kawashima, L., Gomes, L., and Caneva, C. (2020). O ensino remoto durante a pandemia de covid-19: desafios, aprendizagens e expectativas dos professores universitários de educação física.

Leão, G., Lima, M., Castro, L., Junior, E., Pinto, L., and Klautau, A. (2019). Projeto celcom: Um processo de inclusão digital em comunidades isoladas através de redes comunitárias. Anais do Computer on the Beach, pages 666-675.

MEC (2020). Microdados.

Nofitasari, R. and Haryadi, S. (2017). Self-healing mechanism with rsrp measurement in lte network. In 2017 3rd International Conference on Wireless and Telematics (ICWT), pages 144-148. IEEE.

Ratusznei, J., Zanotto, W., and Kamienski, C. (2016). Qualidade de experiência do uusário em uma rede wifi municipal usando streaming de vídeo. In Anais do XLIII Seminário Integrado de Software e Hardware, pages 83-94. SBC.

Rotas, E. E. and Cahapay, M. B. (2020). Difficulties in remote learning: Voices of philippine university students in the wake of covid-19 crisis. Asian Journal of Distance Education, 15(2):147-158. 
Xiong, J., Lipsitz, O., Nasri, F., Lui, L. M., Gill, H., Phan, L., Chen-Li, D., Iacobucci, M., Ho, R., Majeed, A., et al. (2020). Impact of covid-19 pandemic on mental health in the general population: A systematic review. Journal of affective disorders.

Zelezny-Green, R., Vosloo, S., and Conole, G. (2018). Digital inclusion for low-skilled and low-literate people: a landscape review. Geneva, Switzerland: United Nations Educational, Scientific and Cultural Organization. 